\title{
Desconstruindo a política cien- tífica no Brasil: evolução da descentralização da política de apoio à pesquisa e inovação
}

Antonio Botelho ${ }^{1}$ Mariza Almeida ${ }^{2}$

Resumo: $\mathrm{O}$ artigo analisa a trajetória e impacto institucional regional das duas principais políticas federais descentralizadas de apoio à ciência, tecnologia e inovação (CT\&l): uma voltada para cooperação universidade-indústria (PAPPE) e outra para o financiamento à inovação nas MPEs (PAPPE Subvenção). Ele explora a aprendizagem de política pública das unidades da federação e de instituições regionais parceiras, bem como a transformação de seus arranjos institucionais e estruturas de governança. A pesquisa conclui que a rápida ascensão da política de inovação e as exigências estruturais e de processo que se impuseram ao Estado brasileiro, alteraram radicalmente o escopo, a forma e a função da política federal de apoio à pesquisa, contribuindo para a desconstrução da política centralizadora de CT\&I.

Política Pública, Inovação, Descentralização, Fomento CT\&I, Regionalização

\section{Introdução}

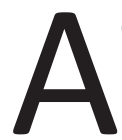

crescente importância da inovação tecnológica na agenda de desenvolvimento econômico e social do país, o reconhecimento de que uma parte importante da cadeia de inovação possui um caráter local (AGRAWAL et al., 2012; BRESNAHAN et al., 2001; SAXENIAN, 1996) e de que a disponibilização de recursos humanos qualificados constitui-se em um dos principais vetores para a inovação (KUZNETSOV, 2006; SAXENIAN, 2006) fazem com que a descentralização das políticas de apoio à ciência, tecnologia e inovação assumam um caráter premente e crítico.

Ao longo das duas últimas décadas, movimentos de descentralização das atividades de fomento federal à ciência, tecnologia e inovação (CT\&l), vêm ocorrendo com o estabelecimento de diversas parcerias entre instituições federais e estaduais. Observa-se tanto a promoção de ações voltadas à
1. Professor Titular, luperj-UCAM, Rio de Janeiro. Email: ajjbotelho@ gmail.com

2. Professora Adjunta, Unirio, Rio de Janeiro Email:

almeida.mariza@ globo.com 
3. Este artigo originou-se de dois estudos realizados pelos autores, em colaboração com Luiziana Aragão, para o Centro de Gestão e Es tudos Estratégicos. O primeiro "Estudos de Caso de Programas Financiados com Recursos dos Fundos Setoriais de CT\&I: PAPPE \& PPP", em 2008. O objetivo do estudo foi identificar e mapear os mecanismos de gestão adotados pelos estados e promover uma análise exploratória dos resultados e impactos preliminares de programas federais descentralizados para os estados (PAPPE e PPP). O segundo, "Programas PAPPE e PAPPE Subvenção - Condições Institucionais e Características de Distribuição (regional, estadual e intraestadual)", executado em 2009. Os autores agradecem o apoio da equipe do CGEE e as críticas e comentários de outros participantes do projeto, em particular Antonio Carlos Galvão, Sofia Daher, Mariano Macedo, Fernanda Sobral e Elizabeth Balbachevsky. descentralização do fomento, quanto à desconcentração dos investimentos públicos federais. O aumento dos investimentos estaduais como contrapartida é uma característica comum a vários desses programas.

A criação dos fundos setoriais mudou a composição das formas de financiamento e representou um incremento no volume de recursos para investimento, uma fonte estável e diversificada, para a CT\&I (MULLER, 2007). O estabelecimento de percentual mínimo de $30 \%$ de investimento dos fundos setoriais nas regiões Norte, Nordeste e Centro Oeste, visando a diminuição das disparidades regionais, representou um movimento importante de desconcentração. Ademais, várias ações financiadas com esses recursos passaram a ser descentralizadas, parcialmente, na sua gestão e implementação, pela Financiadora de Estudos e Projetos (FINEP) e pelo Conselho Nacional de Desenvolvimento Científico e Tecnológico (CNPq), instituições de fomento vinculadas ao Ministério da Ciência, Tecnologia e Inovação (MCTI), e para os estados, por intermédio, principalmente, das Fundações Estaduais de Amparo à Pesquisa (FAP).

São objeto do presente artigo algumas das primeiras experiências da parceria entre governos federal e estaduais para o fomento da inovação, com recursos dos fundos setoriais, realizadas por intermédio da FINEP e das FAP. Seu objetivo é explorar as consequências da descentralização do fomento em CT\&I para a aprendizagem de política pública no Estado e nas unidades federativas, e suas instâncias regionais parceiras, assim como as transformações decorrentes nas suas estruturas institucionais e arranjos de governança. Para tal, é realizada uma análise empírica multidimensional da implementação e dos resultados de dois programas do governo federal: Programa de Apoio à Pesquisa em Empresas (PAPPE) e seu sucedâneo Programa de Apoio à Pesquisa em Empresas na Modalidade Subvenção a Micro e Pequenas Empresas, PAPPE Subvenção ${ }^{3}$. Ela explora ainda, nos limites desses dois estudos de caso, isoladamente e no seu conjunto, seus impactos sobre a desconcentração das ações e instrumentos empregados pelos parceiros estaduais e, principalmente, sobre a escala e escopo dos programas, bem como em suas dimensões geopolíticas espaciais (nos níveis regional, estadual e municipal).

Este artigo está dividido em cinco seções, incluindo a presente introdução. A segunda compreende o marco analítico e a metodologia. A terceira apresenta a análise de dados da pesquisa de campo. A quarta discute os resultados. A quinta seção apresenta conclusões e recomendações.

\section{Marco analítico e metodologia}

O artigo parte da premissa de que uma análise empírica do processo de 
descentralização complementa, valida e joga luz em novos aspectos do fenômeno, para além dos limites naturais de uma análise estruturalista estática e retrospectiva baseada no mapeamento e tipologização de programas e de uma recuperação da linha do tempo realizada até então (CGEE, 2010). Pois é, primeiro, na análise da prática da implementação dos programas e de seus efeitos nas dimensões selecionadas que se evidenciam a diversidade de experiências que alimentam os aprendizados institucionais críticos para e evolução sustentável da política de descentralização. Segundo, são os resultados dessa análise e da avaliação das dimensões que informam as estratégias institucionais e moldam as diretrizes das trajetórias das políticas e as motivações e objetivos dos parceiros que, por sua vez, revelam pistas e marcos para a reorientação eficaz das políticas de descentralização do Governo Federal. Isso ocorre por meio da redefinição de seus instrumentos, mecanismos, governança e práticas de um relacionamento confiante, com vistas à melhoria contínua da eficácia da política nacional de CT\&I.

O marco analítico adota os seguintes conceitos apresentados em CGEE (2010):

- Descentralização se refere à distribuição de funções entre diferentes níveis de governo (federal, estadual e municipal) e entre esses níveis e instituições do setor privado. O processo de descentralização possui duas dimensões analíticas: a descentralização de competências e a descentralização da autoridade decisória. A análise do processo de descentralização permite desagregar os papéis desempenhados pelos diferentes níveis de governo e instituições do setor privado, bem como seus efeitos sobre a coordenação de políticas. Variadas combinações dessas diferentes dimensões do processo podem constituir distintos padrões ou modelos de coordenação federativa e público-privada do fomento em CT\&l;

- Desconcentração se refere às iniciativas do Governo Federal (ministérios, empresas públicas, institutos de tecnologia, etc.), que levam a uma maior dispersão espacial de seus programas de CT\&l, sem que necessariamente estejam associadas à processos de descentralização ou a alguma forma de articulação estratégica, institucional ou operacional específica com as demais Unidades da Federação. De forma semelhante, se refere a iniciativas dos estados e municípios, sem que necessariamente estejam associadas a processos de descentralização ou a alguma forma de articulação estratégica, institucional ou operacional específica com o Governo Federal.

O estudo acadêmico de processos de descentralização da política pública para ciência, tecnologia e inovação tem sido objeto de uma literatura crescente a nível mundial (FRITSCHA \& STEPHANB, 2005; NISCHALKE \& SCHÖLLMANN, 2005). No Brasil, entretanto, essa literatura ainda é escassa e incipiente. 
Balbachevsky (2008), em sua resenha da literatura, conclui que:

Uma das dimensões presentes no debate contemporâneo sobre as reformas das políticas públicas em diferentes áreas diz respeito à efetividade dos esforços para sua regionalização. Para muitos autores, um dos pontos fortes da descentralização decorre do fato de que esse processo permite a emergência e organização de demandas locais que, de outra maneira, permaneceriam desatendidas por políticas desenhadas a partir de iniciativas do governo central. Entretanto, esse resultado não é assegurado pela simples regionalização de políticas cujo desenho permanece sobre o controle do poder central. Ao contrário, como argumentam Tödtling e Trippl (2005), o sucesso da desse processo é criticamente dependente da flexibilização do seu desenho, o que permite sua adaptação para a realidade local.

Nesse sentido, uma hipótese que informa a pesquisa é que a flexibilidade no desenho e implementação de programas de descentralização de fomento federal é uma primeira condição necessária, porém não suficiente, para o desenvolvimento de um experimentalismo federativo, fundamentado na prática institucional e organizacional do aprendizado por monitoramento (SABEL, 1995; 1996).

Os dois programas analisados representam diferentes esforços de descentralização, ainda que com objetivos distintos. Tendo em vista que foram lançados sequencialmente, em distintos momentos históricos, eles permitem a realização de uma análise longitudinal preliminar da trajetória evolutiva desses esforços. As pesquisas de campo que alimentaram essa pesquisa foram realizadas em dois blocos, em dois anos diferentes - PAPPE, em 2008, e PAPPE Subvenção, em 2009 -, requerendo, assim, abordagens metodológicas distintas em função da natureza dos programas e de suas práticas diferenciadas de implementação. O marco comparativo proposto dos dois programas está baseado nos seguintes aspectos: alcance e escopo, contextualização financeira, distribuição dos projetos, recursos, e por regiões, estados e, em menor detalhe, municípios. Assim, na perspectiva da política de inovação emergente, enquanto que o PAPPE se desenvolve no limiar do lançamento da Lei de Inovação (2004), o seu sucedâneo, PAPPE Subvenção, expressa a busca por uma maior capilaridade daquele que é o programa farol no marco da Lei de Inovação, a Subvenção Econômica, que visa subsidiar as atividades de P\&D e inovação das empresas, cujo primeiro edital foi lançado em 2006.

No que tange a metodologia de pesquisa adotada, devido ao pioneirismo dos estudos empíricos de campo sobre a prática da descentralização da CT\&I no país, foi necessário desenvolver metodologias próprias para realização da 
pesquisa, no período 2008/2009, sobre o programa PAPPE e PAPPE Subvenção4.

Os dados coletados permitiram, por um lado, uma visão geral dos programas que, por sua vez, foi crítica para a definição metodológica da pesquisa de campo. Face à dimensão territorial, o curto horizonte de tempo para a execução do projeto e os recursos disponíveis, arbitrou-se um limite de cinco estados para a pesquisa de campo relativa ao PAPPE. O critério de seleção foi composto por sucessivas regras ou recortes. Uma primeira regra observada foi participação das cinco grandes regiões geográficas do país ( $S, S E, C O, N E, N)$. Em seguida, levando em consideração questões de desenvolvimento institucional e conjuntura, a seguinte lista de estados emergiu: RS, MG, MT, SE e AM, apresentados na Tabela 1.

Tabela 1: Desenvolvimento Institucional

\begin{tabular}{|c|c|c|c|}
\hline Estado & Região & \multicolumn{2}{|c|}{ Desenvolvimento institucional } \\
\hline AM & Norte & Crescente & $\begin{array}{l}\text { Primeira unidade da federação a promulgar uma lei esta- } \\
\text { dual de inovação, em janeiro de } 2006 \text {. A Fapeam tem ex- } \\
\text { perimentado um forte crescimento institucional no seu } \\
\text { número de programas, nos seus recursos próprios e na } \\
\text { captação de recursos federais para investimento em C\&T }\end{array}$ \\
\hline SE & $\begin{array}{l}\text { Nor- } \\
\text { deste }\end{array}$ & $\begin{array}{l}\text { Emergen- } \\
\text { te }\end{array}$ & $\begin{array}{l}\text { Este estado possui um porte econômico pequeno } \\
\text { comparável a uma significativa parcela de estados, } \\
\text { e um volume ainda reduzido de projetos em am- } \\
\text { bos os programas, e por esse último já parecer ter } \\
\text { uma consolidação na sua trajetória de crescimen- } \\
\text { to institucional na área de CT\&l, por meio da Fapitec }\end{array}$ \\
\hline MT & $\begin{array}{l}\text { Centro- } \\
\text { Oeste }\end{array}$ & $\begin{array}{l}\text { Incipien- } \\
\text { te-Evolu- } \\
\text { tivo }\end{array}$ & $\begin{array}{l}\text { A Fapemat experimentou entre } 2002 \text { e } 2004 \text { um } \\
\text { fortíssimo crescimento no número programas de } \\
\text { apoio e no volume total de recursos investidos } \\
\text { (próprios e de convênio). Os recursos de convê- } \\
\text { nio que eram superiores aos próprios não se man- } \\
\text { tiveram nos anos seguintes. Em janeiro de } 2008 \\
\text { o estado sancionou sua lei de inovação estadual. }\end{array}$ \\
\hline MG & $\begin{array}{l}\text { Sudes- } \\
\text { te }\end{array}$ & $\begin{array}{l}\text { Maduro- } \\
\text { Evolutivo }\end{array}$ & $\begin{array}{l}\text { A Fapemig possui um orçamento de recursos pró- } \\
\text { prios para investimento elevado e tem desenvolvido } \\
\text { novos programas e parcerias federais e intraestadu- } \\
\text { ais, adensando seus instrumentos e mecanismos. }\end{array}$ \\
\hline RS & Sul & $\begin{array}{l}\text { Maduro- } \\
\text { Bloque- } \\
\text { ado }\end{array}$ & $\begin{array}{l}\text { O estado enfrentou dificuldades orçamentárias e sua } \\
\text { FAP, Fapergs, sofreu com problemas de governança. As } \\
\text { soluções desenvolvidas para manter o nível de recursos } \\
\text { para CT\&I e as adaptações institucionais para executar } \\
\text { os programas e as parcerias com o governo federal po- } \\
\text { dem oferecer ensinamentos úteis para outros estados e } \\
\text { para o governo central em momentos futuros de crise. }\end{array}$ \\
\hline
\end{tabular}

Fonte: CGEE (2008)
4. Em função da natureza peculiar do estudo, foi fundamental o estabelecimento de colaboração entre o Centro de Gestão e Estudos Estratégicos (CGEE) e a agência federal de fomento, Finep, e o Conselho Nacional das Fundações Estaduais de Amparo à Pesquisa (Confap). Já a coleta de dados nas fundações de amparo à pesquisa (FAP) contou com a parceria efetiva do Confap. 
A pesquisa de campo do PAPPE baseou-se na realização de entrevistas semiabertas com pesquisadores (de ICT ou das empresas), com o objetivo de capturar: a) as motivações para participação no projeto por parte dos gestores, pesquisadores e empresários; b) o aprendizado institucional dos diferentes agentes; c) as adicionalidades geradas pelos programas nos dispêndios locais; d) as apreciações de resultados e de impacto; e) os problemas e obstáculos identificados por executores e coordenadores de projetos e seus parceiros e gestores locais em relação aos programas. A seleção de projetos por estado, para as entrevistas, baseou-se no número de projetos, correspondendo a $15 \%$ do seu total.

Com relação ao PAPPE Subvenção, para seleção dos projetos em cada estado, a primeira regra observada foi a de que os estados selecionados estivessem incluídos entre os 5 estados objeto da fase anterior. Uma segunda regra estabelecida foi que, entre as instituições parceiras aprovadas na Chamada Pública realizada pela FINEP, estivesse presente uma proposta cuja execução fosse dirigida por uma FAP e outra com um arranjo institucional diferenciado, a fim de analisar, de forma exploratória, essa diversidade de governança até então não observada em projetos de apoio à inovação. Seguindo essas regras, foram escolhidos os estados de Minas Gerais e o Rio Grande do Sul. Para seleção das empresas a entrevistar foi estabelecida uma porcentagem de $25 \%$ dos projetos, nos quatro municípios com maior número de empresas aprovadas. Em ambas componentes, buscou-se também realizar entrevistas com gestores desses programas nas agências federais, para capturar seus diagnósticos, justificativas, objetivos, aprendizados, motivações e avaliações de resultados, na perspectiva da descentralização.

\section{Descentralização na operacionalização do PAPPE e PAPPE Subvenção}

\section{PAPPE}

O PAPPE foi criado em 2004, num momento de discussão da Lei de Inovação, e da transição sobre a forma de organização e de execução da pesquisa de interesse das empresas, bem como, da destinação dos recursos públicos para esse tipo de atividade. Ele tem por objetivo, dentre outros, estimular a associação de pesquisadores a empresas de base tecnológica em projetos de inovação tecnológica, com vista a valorizar a atividade de pesquisa, desenvolvimento e inovação em ambientes empresariais. O programa, até 2005 , ano de seu último edital, havia investido em torno de $\mathrm{R} \$ 87$ milhões e sido implementado em 19 unidades da federação; ele tinha uma previsão de investimento mínimo por parte dos estados, na mesma proporção, perfazendo um total de $\mathrm{R} \$ 170$ milhões. O valor individual máximo dos projetos deveria respeitar os limites da 
FINEP para cada etapa (Fase I, R\$ 50.000,00; Fase II, R\$ 150.000,00). Ao critério de cada FAP, em decorrência de contrapartida própria ou de terceiros envolvidos nos projetos, esse valor podia ser elevado. Como programa de transição que foi o PAPPE, na sua primeira edição destinava o recurso a pesquisadores, fossem estes ligados a ICT/IES ou da própria empresa.

A Tabela 2, abaixo, apresenta a distribuição dos projetos e valores aprovados para as 18 FAP participantes da pesquisa (de 17 estados e do Distrito Federal). Observa-se que a distribuição regional dos recursos do PAPPE às regiões Nordeste e Norte, em conjunto, é de $28 \%$, ligeiramente inferior ao piso obrigatório de $30 \%$ para distribuição dos recursos dos fundos setoriais para as regiões Norte e Nordeste. As UF com os maiores valores aprovados são: RJ, RS, MG, BA e CE. As UF com os três maiores valores aprovados representam $48 \%$ e as cinco maiores representam $65 \%$ do total.

Tabela 2: PAPPE - Número de projetos e valor aprovado

\begin{tabular}{|c|c|c|c|}
\hline \multirow[t]{2}{*}{ INSTITUIÇÃO PROPONENTE } & \multicolumn{3}{|c|}{ PAPPE Subvenção } \\
\hline & UF & № PROJETOS & VALOR \\
\hline \multicolumn{4}{|l|}{ Região Norte } \\
\hline $\begin{array}{l}\text { Fundação de Amparo à Pesquisa do Estado do } \\
\text { Amazonas (FAPEAM) }\end{array}$ & AM & 23 & $2.832 .881,00$ \\
\hline $\begin{array}{l}\text { Fundação de Amparo à Pesquisa do Estado do Pará } \\
\text { (FAPESPA) }\end{array}$ & PA & 22 & $1.945 .482,98$ \\
\hline Sub total & 2 & 45 & $4.778 .363,97$ \\
\hline Valor médio por projeto & & & $106.185,86$ \\
\hline \multicolumn{4}{|l|}{ Região Nordeste } \\
\hline $\begin{array}{l}\text { Fundação Cearense de Desenvolvimento Científico e } \\
\text { Tecnológico (FUNCAP) }\end{array}$ & CE & 35 & $6.316 .763,72$ \\
\hline $\begin{array}{l}\text { Fundação de Apoio à Pesquisa do Estado do Rio } \\
\text { Grande do Norte (FAPERN) }\end{array}$ & $\mathrm{RN}$ & 8 & $995.653,79$ \\
\hline $\begin{array}{l}\text { Fundação de Amparo à Ciência e Tecnologia do Estado } \\
\text { de Pernambuco (FUNCEPE) }\end{array}$ & PE & 20 & $3.252 .320,00$ \\
\hline $\begin{array}{l}\text { Fundação de Amparo à Pesquisa do Estado da Bahia } \\
\text { (FAPESB) }\end{array}$ & BA & 77 & $10.167 .862,50$ \\
\hline $\begin{array}{l}\text { Fundação de Amparo à Pesquisa do Estado de Alagoas } \\
\text { (FAPEAL) }\end{array}$ & AL & 5 & $941.100,00$ \\
\hline $\begin{array}{l}\text { Fundação de Amparo à Pesquisa e à Inovação } \\
\text { Tecnológica do Estado de Sergipe (FAPITEC) }\end{array}$ & SE & 11 & 1.216.129,97 \\
\hline Sub total & 6 & 156 & $22.889 .829,98$ \\
\hline Valor médio por projeto & & & $146.729,67$ \\
\hline \multicolumn{4}{|l|}{ Região Centro-Oeste } \\
\hline $\begin{array}{l}\text { Fundação de Apoio à Pesquisa do Distrito Federal } \\
\text { (FAPDF) }\end{array}$ & DF & 42 & $5.342 .276,43$ \\
\hline $\begin{array}{l}\text { Fundação de Amparo à Pesquisa do Estado de Mato } \\
\text { Grosso (FAPEMAT) }\end{array}$ & MT & 13 & $864.234,19$ \\
\hline $\begin{array}{l}\text { Fundação de Apoio ao Desenvolvimento do Ensino, } \\
\text { Ciência e Tecnologia do Estado de Mato Grosso do Sul } \\
\text { (FUNDECT) }\end{array}$ & MS & 6 & $521.580,74$ \\
\hline Sub total & 3 & 61 & 6728091,36 \\
\hline Valor médio por projeto & & & $110.296,57$ \\
\hline
\end{tabular}




\begin{tabular}{|c|c|c|c|}
\hline Região Sudeste & & & \\
\hline $\begin{array}{l}\text { Fundação de Apoio à Ciência e Tecnologia do Espírito } \\
\text { Santo (FAPES) }\end{array}$ & ES & 14 & $240.195,00$ \\
\hline $\begin{array}{l}\text { Fundação de Amparo à Pesquisa do Estado de Minas } \\
\text { Gerais (FAPEMIG) }\end{array}$ & MG & 89 & $11.347 .894,36$ \\
\hline $\begin{array}{l}\text { Fundação Carlos Chagas Filho de Amparo a Pesquisa do } \\
\text { Rio de Janeiro (FAPERJ) }\end{array}$ & RJ & 64 & $19.702 .410,00$ \\
\hline $\begin{array}{l}\text { Fundação de Amparo à Pesquisa do Estado de São } \\
\text { Paulo (FAPESP) }\end{array}$ & SP & 20 & $9.654 .564,90$ \\
\hline Sub total & 4 & 187 & $40.945 .064,26$ \\
\hline Valor médio por projeto & & & $218.957,56$ \\
\hline \multicolumn{4}{|l|}{ Região Sul } \\
\hline $\begin{array}{l}\text { Fundação de Apoio à Pesquisa Científica e Tecnológica } \\
\text { do Estado de Santa Catarina (FAPESC) }\end{array}$ & SC & 48 & $3.168 .307,43$ \\
\hline $\begin{array}{l}\text { Fundação de Amparo à Pesquisa do Estado do Rio } \\
\text { Grande do Sul (Fapergs) }\end{array}$ & RS & 62 & $13.926 .449,17$ \\
\hline Fundação Araucária & PR & 53 & $5.762 .755,44$ \\
\hline Sub total & 3 & 163 & $22.857 .512,04$ \\
\hline Valor médio por projeto & & & $140.230,13$ \\
\hline Total & & 612 & $98.198 .861,62$ \\
\hline
\end{tabular}

Fonte: CGEE (2008).

Os dados relativos à MG revelam a existência de um programa local anterior, estruturado pela Fapemig, que aportava recursos para concessão de financiamentos bancários por meio do BDMG. No caso do RS, destacam-se os recursos repassados a empresas incubadas, por meio de incubadoras, que foi um dos instrumentos utilizados por agências federais ou pelo Sebrae, no período anterior ao programa. De forma geral, predominam, ainda, nesses dois estados, os recursos não financeiros disponibilizados para esse tipo de empresa (incubada), por meio de bolsas, sejam por instituições locais ou federais.

A análise dos resultados mostra que as externalidades decorrentes da execução de projetos do PAPPE (outros apoios recebidos financeiros ou não, como por exemplo, bolsas) não alteram significativamente sua distribuição regional, antes da implementação do PAPPE. A maioria dos projetos que receberam outros apoios (de natureza financeira ou não financeira), nos estados analisados, permanecem concentrados em MG e RS. No caso destes, as empresas, após aprovação do projeto PAPPE, tiveram um maior número de apoios financeiros, em contraste com a fase anterior ao PAPPE. Isso pode ser creditado, em parte, à recente diversificação de programas das instituições de fomento, em particular, federais. Anteriormente, os programas de apoio federais e, em particular, os estaduais, se concentravam, primordialmente, na concessão de bolsas. Por outro lado, o relativamente baixo número de eventos parece confirmar resultados de outros levantamentos, como o feito pela FIESP (2007), de que as empresas, particularmente, a micro e pequena, desconhecem, em grande medida, os 
mecanismos de apoio à inovação. Um resultado convergente aqui encontrado foi que o número de empresas que tiveram algum contato ou conhecimento do mecanismo de financiamento pelo capital de risco foi insignificante.

Outro olhar desenvolvido foi focado nas externalidades produzidas pelo PAPPE, baseado no cruzamento daquelas empresas do PAPPE, em seus respectivos estados, com suas submissões de projetos e aprovações no programa de Subvenção Econômica da FINEP, principal programa federal de apoio não reembolsável às empresas no país, para os anos $2006 \mathrm{e}$ 2007. Os dados indicam uma tendência da capacitação das empresas em alavancar recursos adicionais para pesquisa tecnológica e inovação, após terem projetos apoiados pelo PAPPE.

Isso aponta para um efeito positivo do PAPPE nas empresas e estados, que passaram a aplicar mais e obter maior taxa de sucesso no programa de subvenção econômica. Entretanto, não é possível estabelecer uma relação direta de causa e efeito, na medida em que outros fatores e experiências podem estar também influenciando essa tendência.

\section{PAPPE Subvenção}

O formato do PAPPE, posteriormente, foi substituído, por contratações efetuadas diretamente com as empresas após a Lei de Inovação. Assim, em 2008, foi lançada uma nova edição do PAPPE: Programa de Apoio à Pesquisa em Empresas na Modalidade Subvenção a Micro e Pequenas Empresas, PAPPE Subvenção. A chamada pública do programa visava selecionar arranjos institucionais nos estados que seriam parceiros na sua implementação. Ao seu término, o PAPPE Subvenção deveria destinar R\$ 250 milhões (R\$ 150 milhões da FINEP e R\$ 100 milhões dos parceiros estaduais) ao desenvolvimento de produtos, processos e serviços inovadores em 17 estados, apoiando cerca de 1.500 micro e pequenas empresas, sempre com recursos não reembolsáveis. Os editais de seleção de projetos em cada estado foram independentes, com valores e objetivos estabelecidos de acordo com as características regionais pelos membros do consórcio (em sua grande maioria liderados por FAPs, mas em três casos por outras instituições de apoio à empresas, por vezes em parceria com a FAP estadual). Em linhas gerais, foram beneficiadas empresas com faturamento de até $R \$ 10,5$ milhões ao ano, com financiamentos entre $R \$$ 50 mil e R\$ 500 mil. O PAPPE Subvenção forneceu apoio para custeio de atividades de pesquisa e desenvolvimento e/ou inovação. 
A análise a seguir está baseada nos dados de 11 (onze) estados onde os editais de seleção foram concluídos até a data de realização da pesquisa, apresentados na Tabela 3.

Tabela 3: PAPPE Subvenção - Número de projetos e valor aprovado

\begin{tabular}{|c|c|c|c|}
\hline \multirow{2}{*}{ INSTITUIÇÃO PROPONENTE } & \multicolumn{3}{|c|}{ PAPPE Subvenção } \\
\hline & UF & $\mathrm{N}^{\circ}$ PROJETOS & VALOR \\
\hline \multicolumn{4}{|l|}{ Região Norte } \\
\hline FAPEAM & $\mathrm{AM}$ & 35 & $5.539 .003,02$ \\
\hline Sub total & 1 & 35 & $5.539 .003,02$ \\
\hline Valor médio por projeto & & & $158.257,22$ \\
\hline \multicolumn{4}{|l|}{ Região Nordeste } \\
\hline FUNCAP & $\mathrm{CE}$ & 21 & $8.949 .892,90$ \\
\hline FAPERN & $\mathrm{RN}$ & 34 & $3.359 .192,98$ \\
\hline FUNCEPE & PE & 44 & $14.836 .000,00$ \\
\hline FAPESB & BA & 18 & $6.693 .680,00$ \\
\hline Sub total & 4 & 117 & $33.838 .865,88$ \\
\hline Valor médio por projeto & & & $289.221,07$ \\
\hline \multicolumn{4}{|l|}{ Região Centro-Oeste } \\
\hline FAPDF & DF & 18 & $7.536 .109,01$ \\
\hline Sub total & 1 & 18 & $7.536 .109,01$ \\
\hline Valor médio por projeto & & & $418.672,72$ \\
\hline \multicolumn{4}{|l|}{ Região Sudeste } \\
\hline FAPES & ES & 8 & $1.934 .361,14$ \\
\hline FAPEMIG & MG & 70 & $19.542 .265,56$ \\
\hline FAPERJ & RJ & 57 & $24.000 .000,00$ \\
\hline Sub total & 3 & 135 & $45.476 .626,70$ \\
\hline Valor médio por projeto & & & $336.863,90$ \\
\hline \multicolumn{4}{|l|}{ Região Sul } \\
\hline FAPESC & $\mathrm{SC}$ & 37 & $9.349 .269,65$ \\
\hline $\begin{array}{l}\text { Serviço de Apoio as Micro e Pequenas Empresas no } \\
\text { Estado do Rio Grande do Sul (SEBRAE-RS) }\end{array}$ & RS & 39 & $12.782 .499,08$ \\
\hline Sub total & 2 & 76 & $22.131 .768,73$ \\
\hline Valor médio por projeto & & & $291.207,48$ \\
\hline Total & & 381 & 114.522 .362 \\
\hline
\end{tabular}

Fonte: CGEE (2009).

Foram aprovadas 381 solicitações relativas ao PAPPE Subvenção em termos nacionais. Esse total está subdividido entre 96 municípios em 11 estados, ou seja, apenas $1,7 \%$ dos municípios no país. Surpreendentemente, o maior valor médio por projeto está na região centro-oeste e o menor, novamente como no PAPPE, na região norte, refletindo, em parte, as características dos sistemas de CT\&I e a competitividade das empresas nessas regiões. Na distribuição regional, as regiđes Nordeste e Norte receberam 35\%, fatia somente abaixo daquela do conjunto das regiões Sul e Sudeste, porém ainda superior ao piso obrigatório de 30\% para distribuição dos recursos dos fundos setoriais para as regiões Norte e Nordeste. 


\section{Discussão dos resultados}

1. Níveis de autonomia

Ainda que os programas sejam, muitas vezes, planejados de forma centralizada, observou-se uma autonomia crescente dos gestores estaduais na indicação de temas e áreas estratégicas, bem como na implementação e gestão dos programas, desde as etapas de seleção de projetos, até a contratação. Essa autonomia foi relativa. Por um lado, nos editais estaduais de ambos programas para seleção de projetos de empresas, a FINEP participou do julgamento. Ademais, a análise e a aprovação da prestação de contas dos pesquisadores (PAPPE) e das empresas (PAPPE Subvenção) era de responsabilidade da FINEP. Por outro, as FAP demonstraram possuir alguma flexibilidade. A FAP possuía autonomia para definir em seus editais as rubricas de investimento, faixas de valor dos projetos e prioridade em termos de áreas estratégicas.

\section{Aprendizado Institucional}

A análise dos resultados apresentados permite concluir que o aprendizado institucional e organizacional foi observado nas FAP e também entre suas instituições parceiras. Aqui, o principal aprendizado observado, uma síntese do institucional e do organizacional, tem sido um aprendizado mútuo de uma prática discursiva entre estas e as FAP.

\section{Impacto sequencial dos programas}

Assim, o PAPPE por efeito da natureza de suas demandas de ordem diversa à maioria dos programas anteriores com foco em projetos de pesquisa científica fez com que os estados se capacitassem institucionalmente. Estes e outros programas (Pronex, DCR, etc.) de parcerias federais com os estados estimularam uma atividade de aprendizado e crescimento institucional local, levando estados: 1) a criarem uma FAP; 2 ) a reforçarem e consolidarem suas FAP para a realização das tarefas demandadas pelos programas; e 3) a expandirem e diversificarem o escopo e intensidade de atuação de suas FAP, aumentando, inclusive, o volume de recursos do tesouro estadual para a área de CT\&I. O exemplo maior aqui é o Amazonas, onde a Fapeam multiplicou, em cinco vezes, seu orçamento desde o início de sua participação nos projetos de fomento descentralizado (PPP e DCR), tornando-se, então, a quarta FAP do país em volume de investimentos. Adicionalmente, para realizar tarefas de prospecção e seleção de propostas, particularmente no PAPPE, as FAP, nos estados de SE, MG e RS, se aproximaram e estabeleceram uma prática discursiva com outros atores diversos daqueles geralmente acadêmicos e majoritariamente públicos com que tinham contato até então. 
Deve ser salientada a mudança no ambiente institucional com relação à inovação no Brasil. O PAPPE foi lançado ainda em 2004, um ano antes da regulamentação da Lei de Inovação, sendo caracterizado como um programa de transição do apoio a empresas intermediado pelas IES/ICT e seus pesquisadores para um novo modelo direcionado à empresa. Na esteira da Lei de Inovação muitos estados aprovaram leis de inovação, ainda que somente em poucos existam fundos próprios para investir em programas de subvenção econômica para inovação em empresas. Como exemplo, pode-se citar Minas Gerais e a previsão de criação no Rio Grande do Sul.

\section{Impacto institucional da descentralização}

Os estados com uma comunidade científica pequena e capacidade institucional inicialmente reduzida em sua FAP, porém com uma capacidade de mobilização de recursos do tesouro estadual, foram os principais beneficiados pela descentralização do fomento público federal para CT\&I. Os estados têm buscado experimentar novos arranjos institucionais por meio da implementação de programas próprios de descentralização, tendo adotado estratégias e modelos distintos em função de sua cultura institucional e seu legado político. O RS tem, desde 2004, um programa ambicioso de descentralização intraestadual (PROCOREDES), com base em demandas regionais definidas de baixo para cima, via Programa de Participação Popular; e MG adotou programa de descentralização regional com viés intrassetorial baseado em setores de crescimento definidos pelo Governo do Estado, ainda que levando em conta suas diferentes competências regionais.

\section{Conclusão}

A pesquisa realizou uma análise exploratória de instrumentos e mecanismos de gestão empregados atualmente na área de CT\&I para o desenvolvimento regional, com vistas à formulação de subsídios técnicos para o aperfeiçoamento das políticas de descentralização do fomento público federal. Um aspecto que se sobressai na análise acima é a relação entre descentralização e a evolução institucional. Os resultados obtidos para os programas PAPPE e PAPPE Subvenção, nas duas etapas do projeto de pesquisa, apresentadas aqui de forma sintética, possuem uma robustez analítica. Em que pese terem sido analisados em pesquisa de campo, por amostragem certa, apenas cinco unidades da federação, na análise foram integrados dados produzidos no trabalho de campo com resultados de levantamentos e análises estatísticas dos programas em questão e entrevistas com atores executores, formuladores e tomadores de decisão política.

Por um lado, recentemente, a política de desenvolvimento regional se preocupa cada vez mais com a capacidade de inovação e o desempenho das 
empresas (KAUFMAN \& WAGNER, 2005). Por outro, para cumprir esse objetivo, ela deve se integrar à política de descentralização do apoio a CT\&l, que, por sua vez, para dar continuidade à sua evolução deve incorporar a lógica da inovação regional. Assim, para se avaliar os novos marcos da evolução de uma política regional de inovação, deve-se levar em conta que a multiplicidade de objetivos pode afetar a implementação e desempenho dessa política pública, ao perseguir missões múltiplas e não necessariamente convergentes em seus objetivos, meios necessários, instituições, governança, atores e dinâmicas evolutivas. A inovação requer, sim, ambientes especializados e cooperativos de inovação, um alvo da lei, mas a formação de alianças estratégicas para o desenvolvimento de projetos de cooperação, outro alvo desta, nem sempre é convergente com o processo de inovação para o mercado, que demanda competição e empreendedores. Pois, a formação de ambientes especializados para inovação (BRESNAHAN, GAMBARDELLA \& SAXENIAN, 2001) demanda uma estratégia voltada para a concentração de recursos financeiros, que tem por objetivo a consolidação de recursos humanos altamente especializados e de instituições e arranjos de governança flexíveis, em contradição com a meta de dispersão de recursos para regiões menos desenvolvidas.

Uma conclusão decorrente das análises feitas é que a existência ou não de uma rede estadual de instituições de Ensino Superior e, preferencialmente, com atividades de pós-graduação e/ou uma rede de ICTs estadual, determina o espectro de possibilidades disponíveis para o estado no desenvolvimento de uma política regional focada de CT\&l; e, logo, o potencial de uso estratégico da descentralização do fomento federal pelo governo estadual. Na amostra, nenhum dos cinco estados possui uma rede estadual de IES desenvolvida, sendo, na maior parte dos casos, emergente e embrionária ( $A M, M G, R S$ ) ou não existente (MT, SE). Uma estratégia alternativa adotada por alguns estados é de dar apoio prioritário por meio de programas locais aos pesquisadores de seus ICT estaduais e suas empresas; fortalecendo-as, para que se tornem mais competitivas nos editais nacionais, com projetos de pesquisa voltados para realização de pesquisas e inovações alinhadas com os objetivos econômicos e sociais do estado. MG, pelo contrário, tem uma rede de IES federais hipertrofiada, ainda em expansão, e descentralizada através do estado, contribuindo para a perspectiva de capacidades básicas de ensino e pesquisa no esforço de descentralização intraestadual. A análise da distribuição por tipo de instituição dos recursos da Fapemig, em 2006, mostrou que as 12 IFES no estado, que concentram 90\% dos doutores, recebem quase dois terços destes, enquanto a contribuição do governo federal para o orçamento de fomento da Fapemig gira em torno de 1/3. Ou seja, o governo estadual financia parte da pesquisa e qualificação de pessoal das IFES no Estado. Entretanto, o Estado tem buscado, mais recentemente, recuperar esses investimentos através de um aumento dos recursos do tesouro local para fomento 
em programas de inovação, em programas para áreas tecnológicas e econômicas estratégicas para o Estado e em programas de capacitação de quadros de pesquisa e operação do setor público estadual. Finalmente, grandes estados, como AM e MT, ficam limitados na sua capacidade de fazer uso estratégico do fomento federal, principalmente no que diz respeito à atração e retenção, e o desenvolvimento de jovens doutores, pela ausência de uma rede estadual de IES e de ICTs. Diferentemente dos outros três estados da amostra, em que as IES particulares jogam um papel preponderante na formação de RH e em alguns casos também na pesquisa, particularmente aquela voltada para a inovação (RS).

Em seguida, a análise desvendou uma série de problemas, obstáculos, fraquezas e desafios comuns aos dois programas analisados, que persistem e devem ser objeto de uma reflexão conjunta aprofundada por parte das agências com o MCTI, as FAP e suas associações representativas, consultores e especialistas acadêmicos. Primeiro, há o problema de demora na implementação efetiva, geralmente causada por deficiências organizacionais, buracos institucionais e instabilidade no repasse de recursos do tesouro estadual. No caso do PAPPE, surgiu também o problema da ambiguidade em relação às fronteiras institucionais e aos mandatos de instituições em áreas afins à montante e à jusante do processo de inovação. Finalmente, persistem deficiências localizadas na capacidade analítica nos quadros das FAP e no marco institucional de relacionamento com seus clientes - pesquisadores, empreendedores e empresas - e outros parceiros institucionais - universidades, associações de classe e instituições de governo. Estas fazem com que atividades críticas para a evolução institucional e para o contínuo e sustentável aperfeiçoamento, como o monitoramento e avaliação de programas, ainda não estejam incorporados, nem na cultura organizacional das FAP, nem nos sistemas de incentivos das agências federais. Adicionalmente, dificuldades estruturais básicas, como a ausência ou baixa informatização dos processos subjacentes aos programas em suas diferentes etapas, tanto nas FAP quanto nas agências (estes, mesmo quando existentes, apresentam um baixo grau de transparência e acessibilidade), bloqueiam o próprio lançamento de qualquer monitoramento para aprendizado (SABEL, 1995); geralmente, reduzindo-o a um mero acompanhamento físico-financeiro e controle administrativo, do qual geralmente não se extraem quaisquer aprendizados para a mudança evolutiva.

Aqui, uma recomendação estratégica para a evolução sustentável dos programas e para o marco institucional de descentralização do fomento é a promoção ativa de atividades de monitoramento e avaliação, tanto dos projetos quanto dos programas. No RS, a experiência das empresas ganhadoras do PAPPE, que formaram uma associação (Ajet) para dialogar e levar suas demandas à Fapergs, é um exemplo promissor. Aqui, trocas institucionais, como as que 
ocorreram na montagem de metodologias, instrumentos e marcos analíticos para avaliação de projetos no âmbito do programa PAPPE, entre a Fapergs e a Fapemig, merecem ser objeto de apoio e análise para produção de conhecimento a ser difundido entre as outras FAP.

Finalmente, no seu conjunto, os resultados analíticos alcançados convergem com aqueles de outras experiências nacionais de descentralização da política de apoio a CT\&I. Por exemplo, uma análise da experiência da Nova Zelândia (NISCHALKE \& SCHÖLLMANN, 2005) conclui que:

As principais lições da experiência da Nova Zelândia são a importância do arranjo institucional nas regiões, a necessidade de se customizar iniciativas aos diferentes contextos regionais e percepções acerca do complexo relacionamento entre o desenvolvimento regional e a política regional de desenvolvimento.

Abstract: This article analyses the trajectory and the regional institutional impact of the two main decentralized federal support policies for science, technology and innovation (ST\&I): one aimed at university-industry cooperation (PAPPE) and another towards the MSE innovation financing (PAPPE Subvenção). It explores the learning of public policy on federative units and their regional institutional partners, as well as its transformation in institutional arrangements and governance structures. The research concludes that the rapid growth of innovation policy in the ST\&I agenda and the structural and process requirements it imposed upon the Brazilian State and its federative units radically altered the scope, the form and the function of the federal policy of researching support, contributing to the deconstruction of the centralizing ST\&I policy.

Keywords: Public Policy, Innovation, Decentralization, ST\&I Funding, Regionalization

\section{Referências Bibliográficas}

AGRAWAL, A. K. L.; COCKBURN, I. M.; GALASSO, A.; OETTL, A. (2012) Why Are Some Regions More Innovative Than Others? The Role of Firm Size Diversity (Working Paper 17793). Cambridge, MA: National Bureau ff Economic Research.

BALBACHEVSKY, E. (2008) Federalismo e Políticas de Ciência, Tecnologia e Inovação: especificidade setorial e marcos institucionais na experiência internacional. Nota Técnica. Brasília: Centro de Gestão e Estudos Estratégicos.

BRESNAHAN, T.; GAMBARDELLA, A.; SAXENIAN, A. (2001) "Old Economy'Inputs for 'New Economy' Outcomes: Cluster Formation in the New Silicon Valleys". Industrial and Corporate Change Vol. 10 n. 4, p. 835-860. 
Centro de Gestão em Estudos Estratégicos (2008) Descentralização do Fomento Federal: Estudos de Caso de Programas Financiados com Recursos dos Fundos Setoriais de CT\&I: PAPPE \& PPP. Brasília Centro de Gestão em Estudos Estratégicos.

Centro de Gestão em Estudos Estratégicos. (2009) Programas PAPPE e PAPPE SUBVENÇÃO - Condições Institucionais e Características de Distribuição (regional, estadual e intra-estadual). Brasília: Centro de Gestão e Estudos Estratégicos.

Centro de Gestão em Estudos Estratégicos. (2010) Descentralização do fomento à ciência, tecnologia e inovação no Brasil. Brasília: Centro de Gestão e Estudos Estratégicos.

FRISTCHA, M. \& STEPHANB, A. (2005) "Regionalization of innovation policy Introduction to the special issue". Research Policy, Vol. 34 n. 8, p. 1123-1127.

KUZNETSOV, Y. (ed.) (2006) Diaspora Networks and the International Migration of Skills - How Countries can Draw Their Talent Abroad (WBI Development Studies). Washington, D.C.: The World Bank

NISCHALKE, T. \& SCHÖLLMANN, A. (2005) "Regional Development and Regional Innovation Policy in New Zealand: Issues and Tensions in a Small Remote Country". European Planning Studies, Vol. 13, n. 4, p. 559-579.

OECD (2002) Benchmarking industry-science relationships. Paris: OECD.

PEREIRA, N. M. (2007) "Fundos setoriais no Brasil: um pouco da história". Ciência e Cultura, Vol. 59, n. 4, p. 37-39.

SABEL, C. F. (1995) "Learning by Monitoring: The Institutions of Economic Development" In: N. Smelser and R. Swedberg (eds.), Handbook of Economic Sociology Princeton, NJ: Princeton University Press, p. 137-165.

(1996) A measure of federalism: assessing manufacturing technology centers". Research Policy, Vol. 2 n. 25, p. 281-307.

SAXENIAN, A. (1996) Regional advantage: culture and competition in Silicon Valley and Route 128. Cambridge, MA: Harvard University Press.

(2006) The new Argonauts: regional advantage in a global economy. Cambridge, MA: Harvard University Press. 